\title{
Research Paper: Reliability of Longus Colli and Capitis Muscles Cross Sectional Area Measurement During Cranio Cervical Flexion Test Using Ultrasonography
}

\author{
Maryam Zargoosh ${ }^{1},{ }^{*}$ Mohsen Amiri ${ }^{1}$, Iraj Abdollahi ${ }^{1}$, Leila Rahnama ${ }^{1}$, Rezvan Lak ${ }^{1}$
}

1. Department of Physiotherapy, University of Social Welfare and Rehabilitation Sciences, Tehran, Iran.

Citation: Zargoosh M, Amiri M, Abdollahi I, Rahnama L, Lak R. [Reliability of Longus Colli and Capitis Muscles Cross Sectional Area Measurement During Cranio Cervical Flexion Test Using Ultrasonography (Persian)]. Archives of Rehabilitation. 2017; 18(1):43-50. https://doi. org/10.21859/jrehab-180143

\section{https://doi.org/10.21859/jrehab-180143}

Received: 11 Oct. 2016

Accepted: 12 Jan. 2017

Keywords:

Reliability, Longus

Colli, Longus capitis, Ultrasonography,

Craniocervical

flexion test

\section{ABSTRACT}

Objective Neck muscles provide more than $80 \%$ of the mechanical stability of the cervical spine. Deep neck flexor muscles including Longus Colli (LC) and longus capitis (LCA) play an important role in maintaining the cervical spine stability. Patients with chronic neck pain suffer from weakness and atrophy of neck flexor muscles. Ultrasonography measurement of muscle dimensions provides the ability to objectively assess muscle atrophy or hypertrophy. Therefore, the aim of this study was to evaluate the reliability of measurement of the cross-sectional area of LC and LCA muscles in five stages of craniocervical flexion (CCF) test utilizing ultrasonography.

Methods \& Materials A total of 10 individuals including 5 patients suffering from chronic nonspecific neck pain and 5 healthy controls voluntarily participated in this study. The cross-sectional areas of LC and LCA muscles were measured at the level of thyroid cartilage during five stages while the subjects performed CCF test using a real-time ultrasound device. Two images were taken on the same day with an hour interval to assess the within-day reliability, and another image taken a week later to determine between-day reliability. SPSS was used to analyze data.

Results The ICC for within-day and between-day reliability in healthy individuals and patients were 0.91 , 0.88 and $0.90,0.86$, respectively. The average standard error of measurement with the minimum of 0.03 and the maximum of 0.05 was obtained.

Conclusion Ultrasonography is a reliable method to measure the cross-sectional area of LC and LCA muscles both in healthy individuals and patients with chronic neck pain during five stages of the CCF test.

\section{* Corresponding Author:}

Mohsen Amiri, PhD

Address: Department of Physiotherapy, University of Social Welfare and Rehabilitation Sciences, Koodakyar Ave., Daneshjoo Blvd., Evin, Tehran, Iran. Tel: +98 (912) 2302813

E-Mail: posturalclinic@yahoo.com 


\title{
تكراريذيرى اندازهيَي سطح مقطع عضالات لونكَوس كولى و لونَّوس كيبتيس هنَّام آزمون كرانيوسرويكال فلكشن توسط اولترئراسونو مفطح عضرافى
}

\author{
مريم زركوش'، "محسن اميرى'، ايرج عبدالهى'، ليلا رهنما'، رضوان لك' \\ 1. أكروه فيزيوترايى، دانشكاه علوم بهزيستى و توانبخشى، تهران، ايران.
}

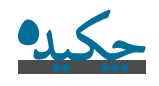

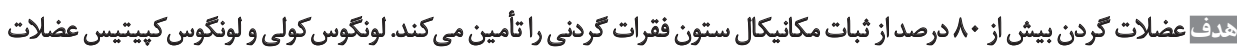

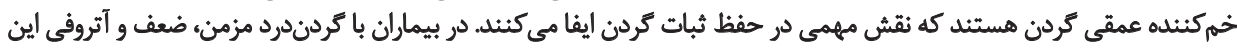

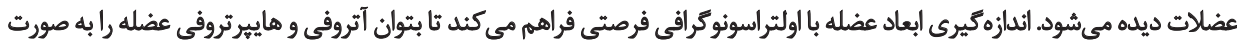

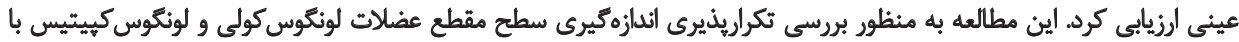

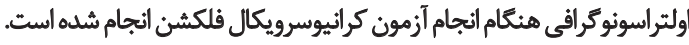

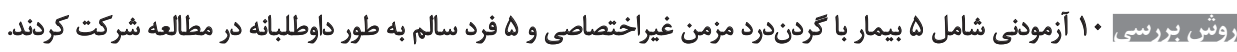

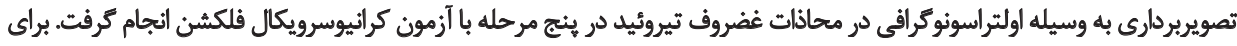

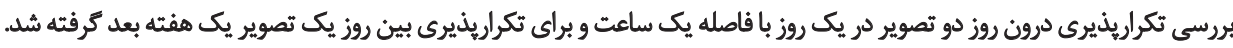

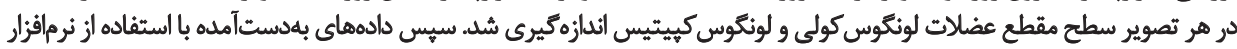

SPSS S تحليل شد.

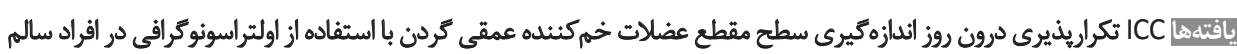

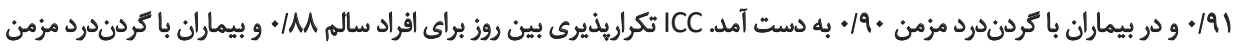

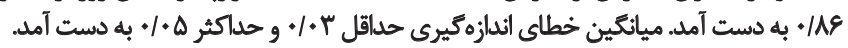

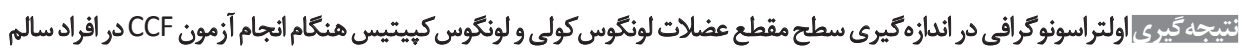
وافراد با كردندرد مزمن غيراختصاصى روشى بائاريا وتكراريذير است.

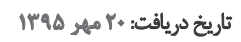

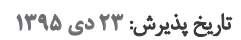

عضله لونكوس كولى از روى زوائد عرضى مهره بهاي سوم، جهارم و

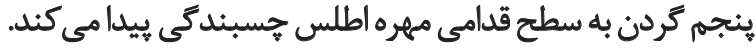
بخش ميانى يا بخش عمودى كه از روى سطح قدام تنه سه مهره

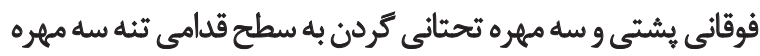

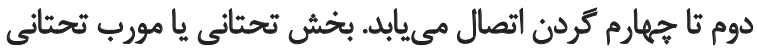

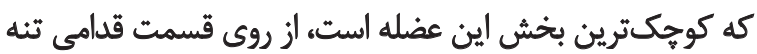

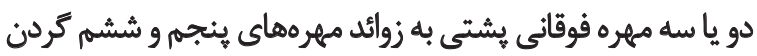

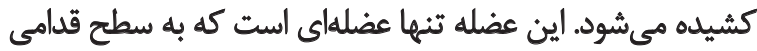

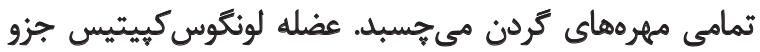

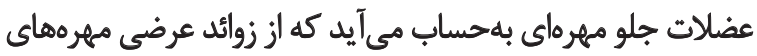

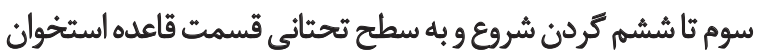

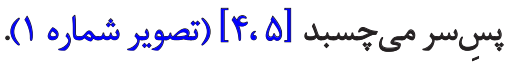

dalä در ستون فقرات، عضلات بههمراه عناصرغير فعال وغيرانقباضى

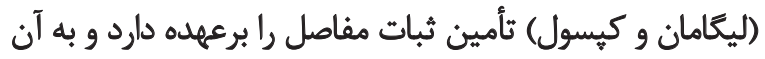

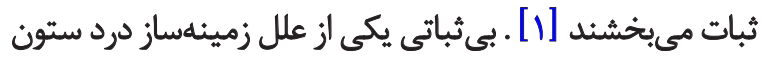

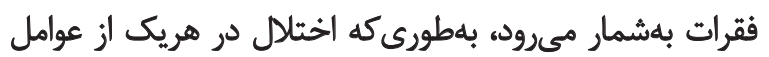

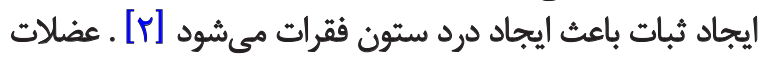

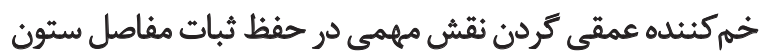
فقرات كردنى ايفا مى كند.

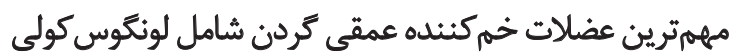

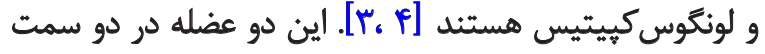

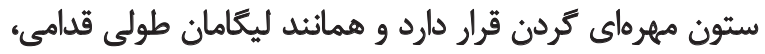

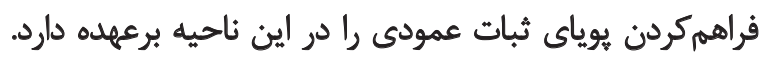

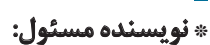

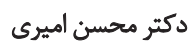

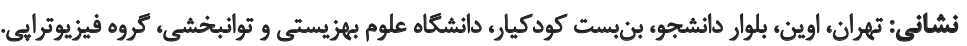

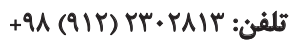
posturalclinic@yahoo.com 
دادند اندازهكيرى ابعاد عضله لونكّوس كولى در افراد سالم و بيماران

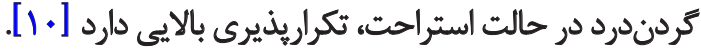

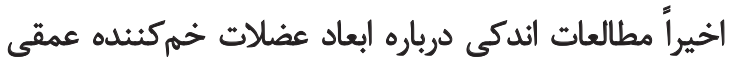

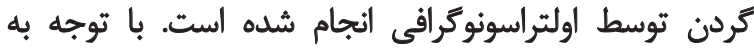

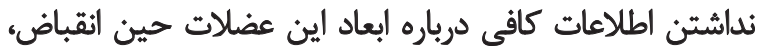

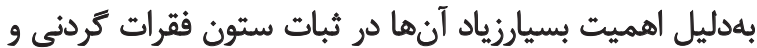

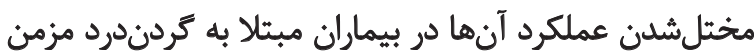

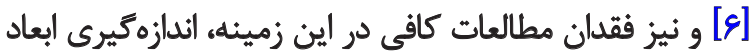

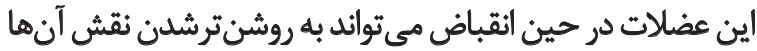

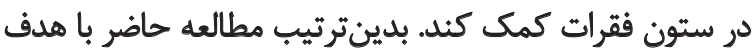

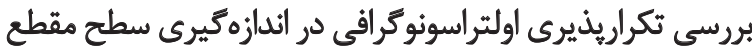

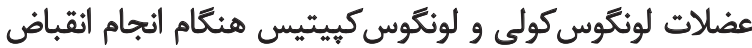

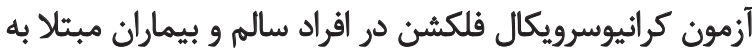
كردندرد مزمن انجام شده است.

$$
\text { روش بروشى }
$$

• آزمودنى (\& زن و \& مرد) شامل ه بيمار مبتلا به كردندرد

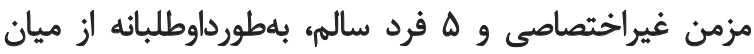

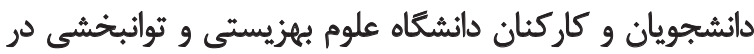

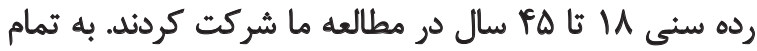

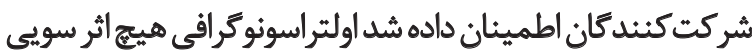
بر سلامتى آنها ندارد.

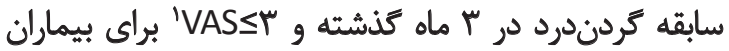

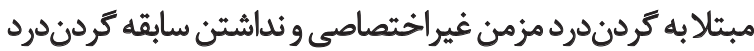

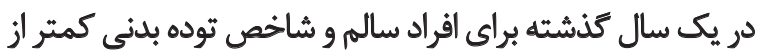

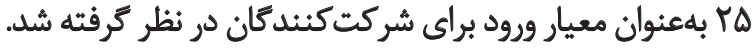

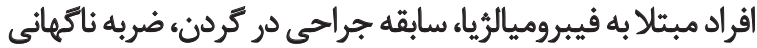

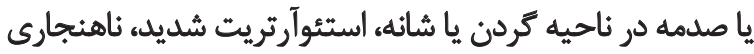

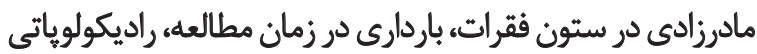

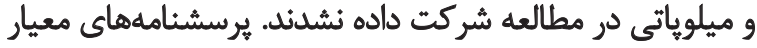

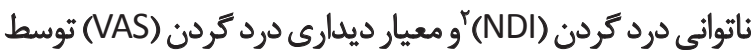

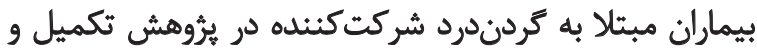

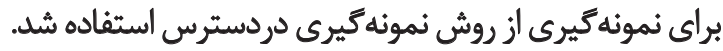
براي اندازهكيري سطحمقطع عضلات ازدستكاهاولتراسونوكرافي

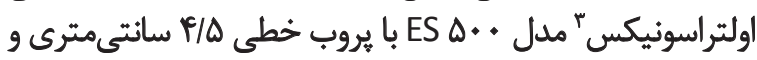

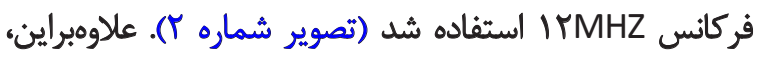

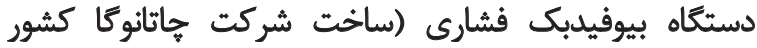

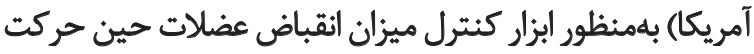

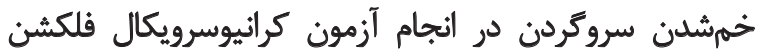
(CCF) مورداستفاده قرار كرفت (تصوير شماره "َّ).

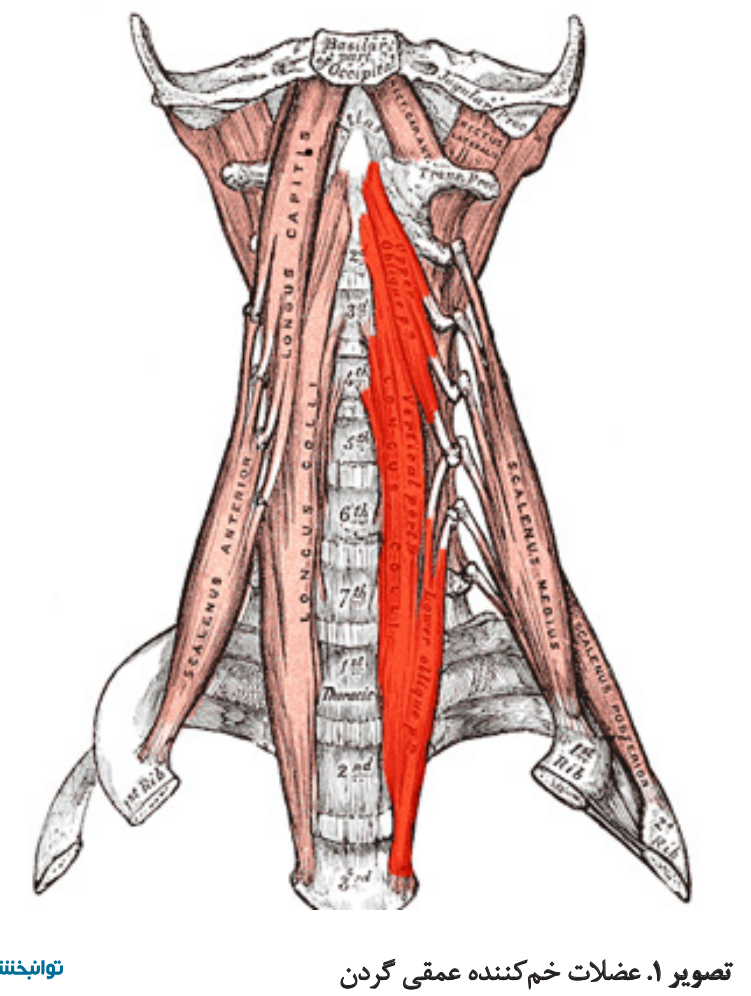

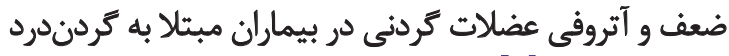

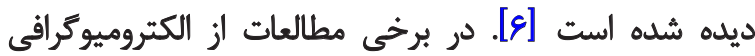

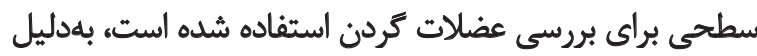

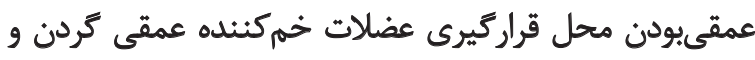

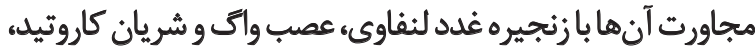

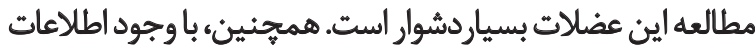

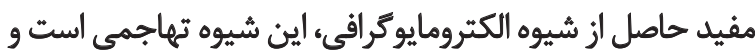

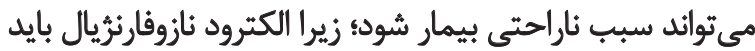

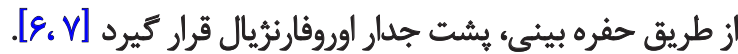
با توجه به اينكه اندازميرى ابعاد عضله معيار ارزشمندى براي

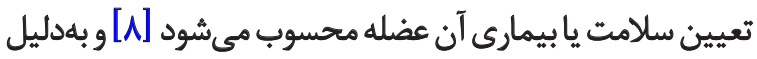

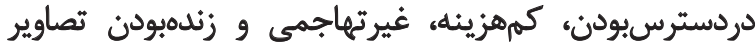

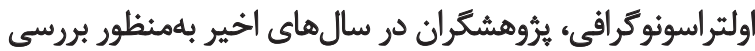

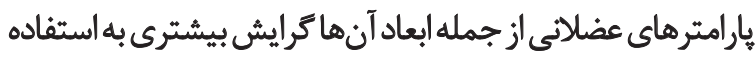

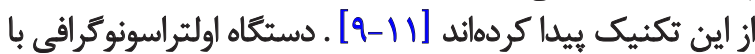

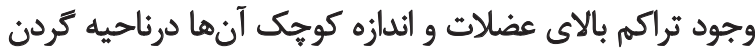

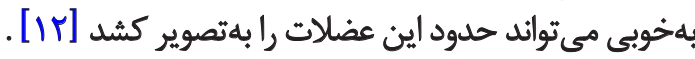
محققان از طريق اولتراسونوگرافى، اطلاعاتى در مورد سطح

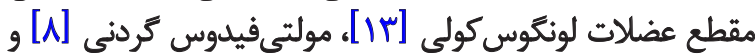

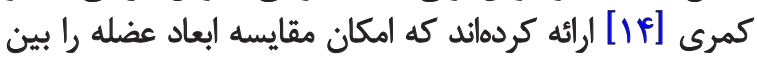

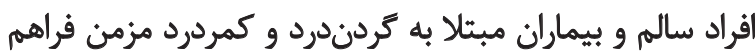
مىسازد. همجنين محققان با استفاده از اولتراسونوكرافي نشان 

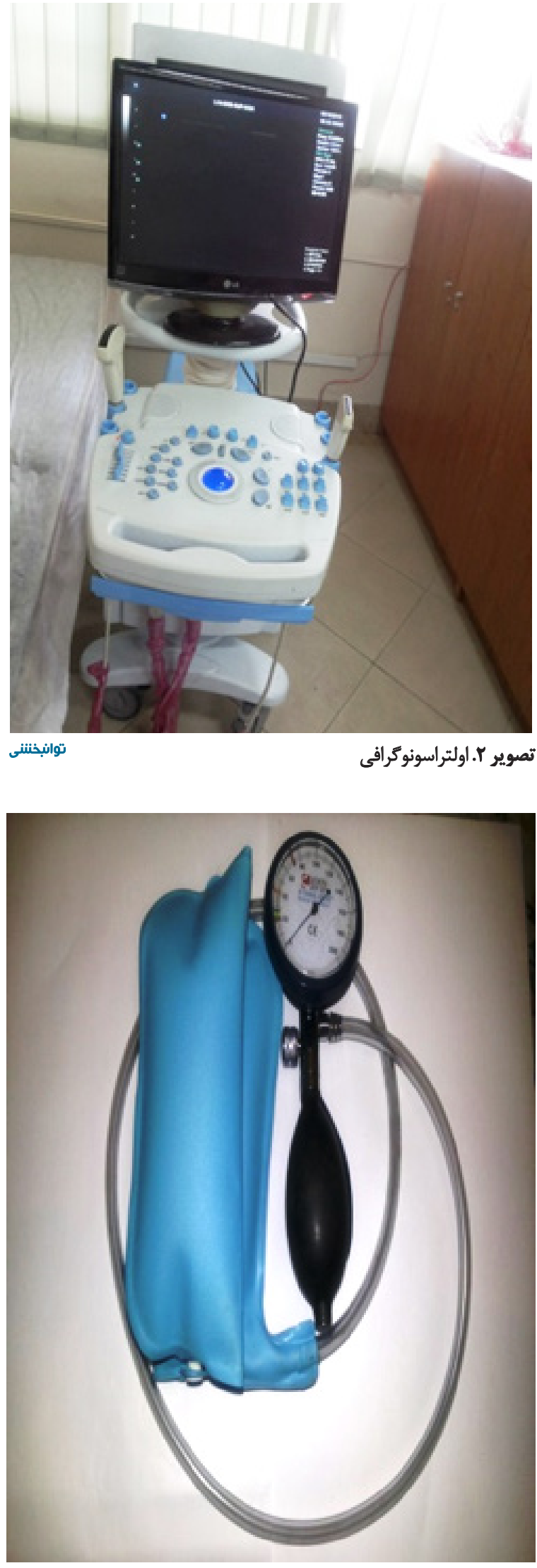

توانبخننى

تصوير ץ. بيوفيدبك فشارى

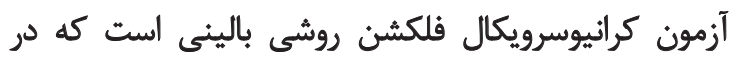

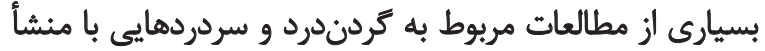

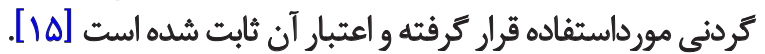

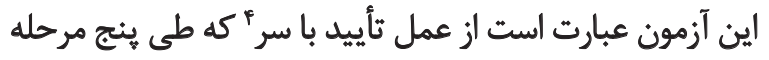

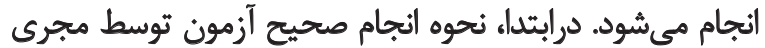

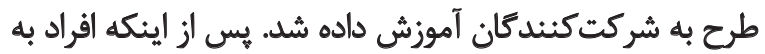

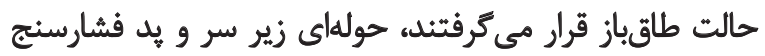

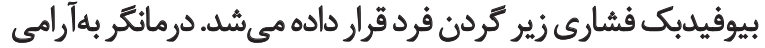

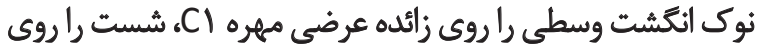

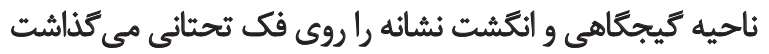

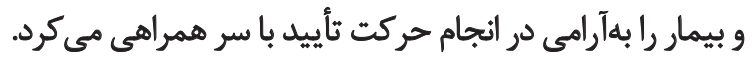
نمايشكر فشارسنج بيوفيدبك طورى قرار مى كرفت كه درمانكر

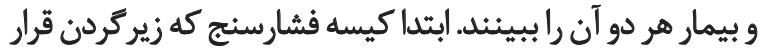

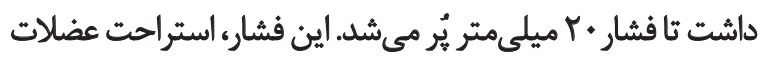

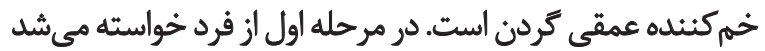

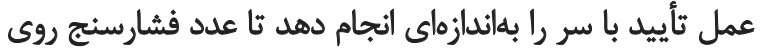

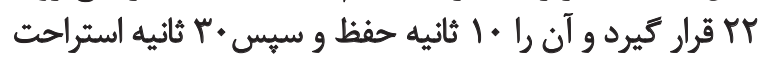

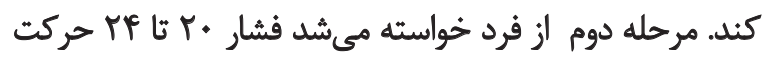

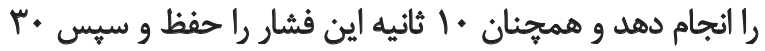

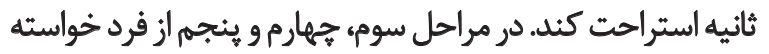

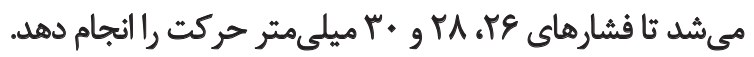
براي انجام تصويربردارى از سطح مقطع عضلات همزمان با بان

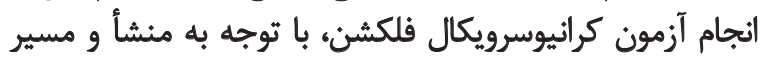

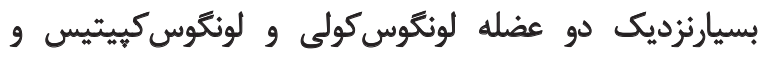

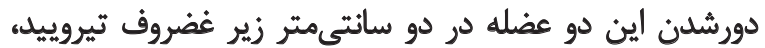

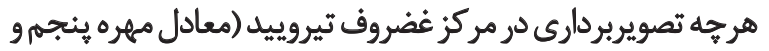

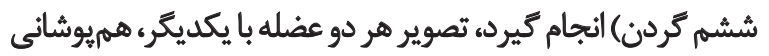

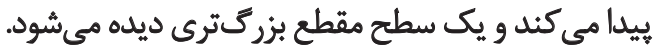

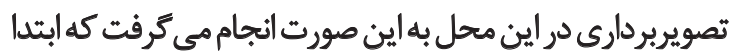

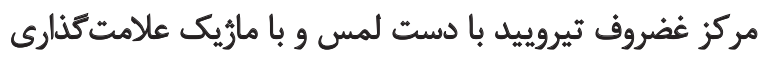

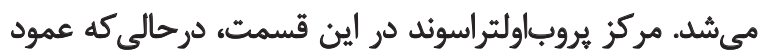

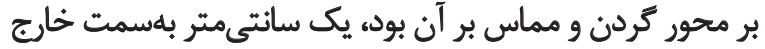

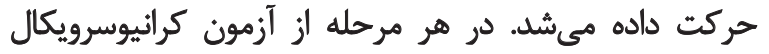

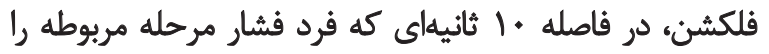

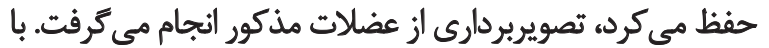

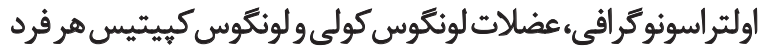

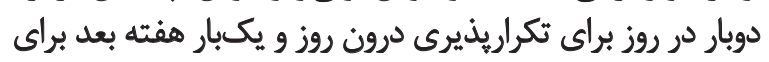

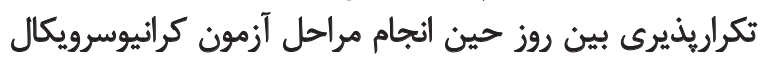
فلكشن اندازهيرى مي بـشد. براى بررسى تكراريذيرى دادها، شاخصهاى ICCه و خطاى 


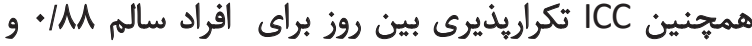

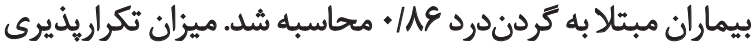

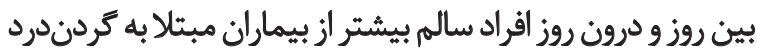

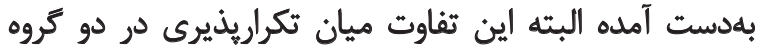

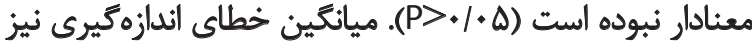

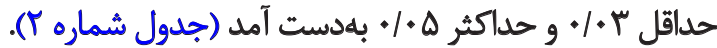

بحث

در مطالعه حاضر نشان داده شد دادههاى حاصل از ارزيابى

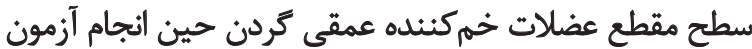

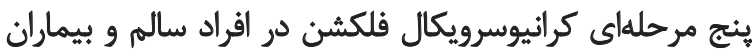

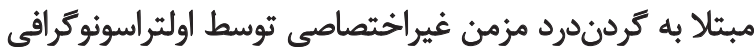

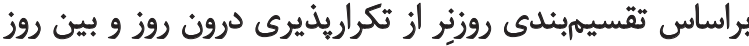

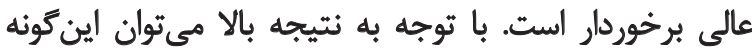

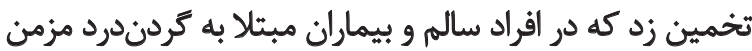

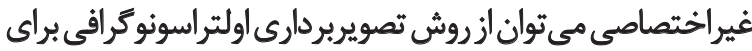

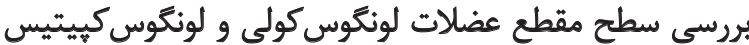
حين انجام انقباض آنها بهره برد

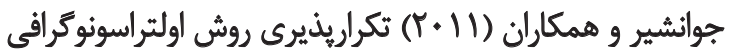

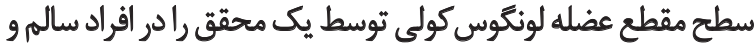

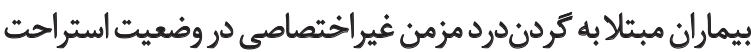

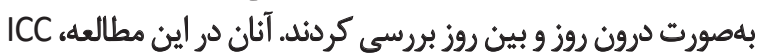

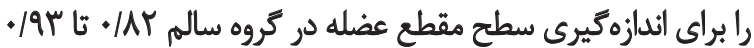

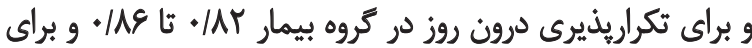

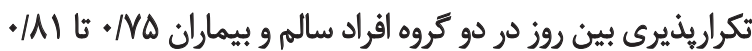

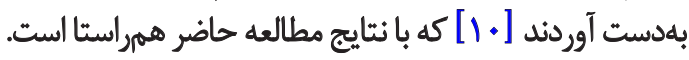

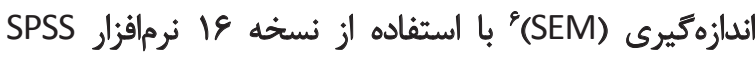

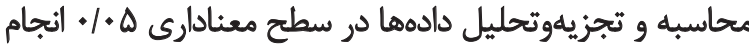
شد. فرمول محاسبه خطاى اندازهيرى بلهشرح زير است:

\section{$S E M=S D V 1-I C C$}

مطلوبيت تكراريذيرى مطالعه ما براساس تقسيم بندى

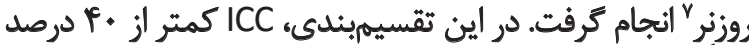

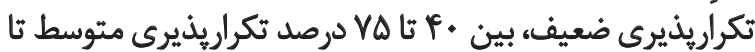

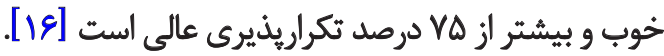

يافتهها

ويرُّكىهاى جمعيتشناختى افراد شامل سن، قده، وزن و

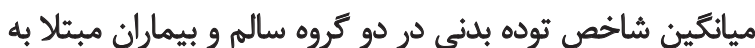

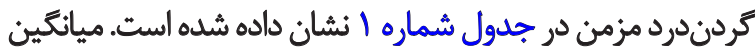

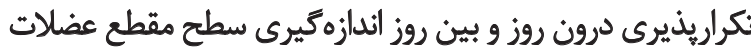

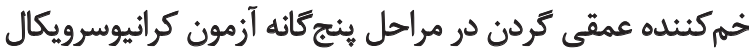

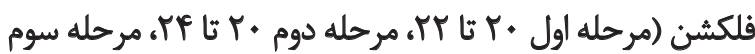

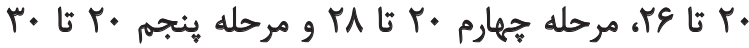

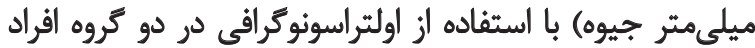

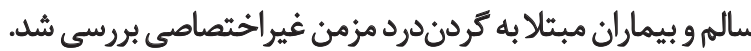
هر آزمون در هر مقطع، ب بار انجام و ميانكين آن براى اندازه

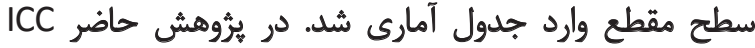

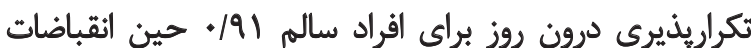

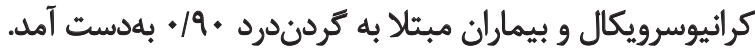

6. Standard Error of Measurement (SEM) 7. Rosner

جدول ا. ويثَّى هاى جمعيتشناختى افراد در دو كروه سالمه و بيماران مبتلا به كردندرد مزمن غيراختصاصى

\begin{tabular}{|c|c|c|c|c|}
\hline بديانىين ثانحراف معيار & ميانكين وزن \$|ثحراف معيار & ميانكين قد土|نحراف معيار & ميانكين سند|نحراف معيار & كروه \\
\hline$M / \Delta A \pm V / 81$ & $8+ \pm 1 \cdot / 48$ & 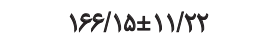 & $M R / A \cdot \pm M / T r$ & اقراد سالم \\
\hline$r / T+ \pm T / T r$ & $\Delta V / \Delta+ \pm \Delta / M$ & $\mid q \Delta / \cdot \Delta \pm q / \gamma^{\prime}$ & $\Gamma \Delta / \cdot \Delta \pm \Psi / Q 1$ & بيماران \\
\hline
\end{tabular}

توانبخننىى

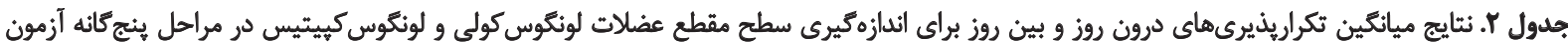
كرائيوسرويكال فلكشن

\begin{tabular}{|c|c|c|c|}
\hline SEM & ICC & تكراريذيرى & L \\
\hline$+1 . r$ &.$/ 91$ & درون روز & \multirow{2}{*}{ الفراد سالم } \\
\hline$+\%$ & $\cdot / M$ & بين روز & \\
\hline $1+\%$ & $* / 9$. & درون روز & \multirow{2}{*}{ بيماران مبتلا به كردندرد مزمن } \\
\hline$+1+\Delta$ & $+\mathbb{N}$ & بين روز & \\
\hline
\end{tabular}

توانبخننى 
غيراختصاصى در مطالعه شركت داشتند، بيشنيهاد مي دشود

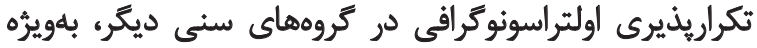

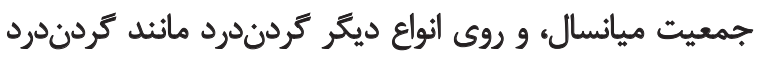

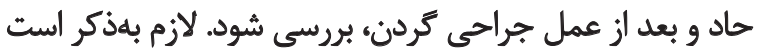

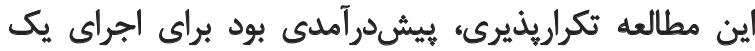

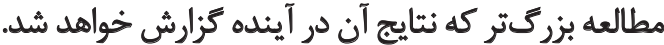

$$
\text { تشكر وقدردانى }
$$

اين مقاله بركرفته از إياياننامه مقطع كارشناسى ارشد مرئم مريم

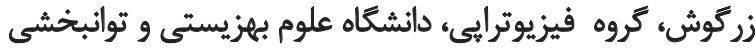

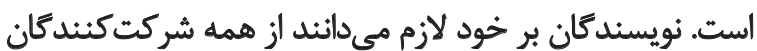
در اين مطالعه تشكر و قدردانى نمايند.

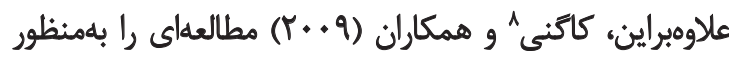

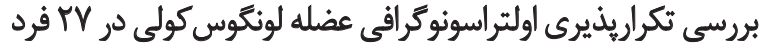

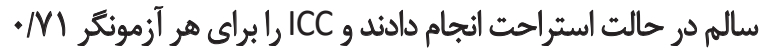

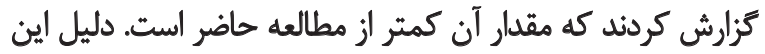

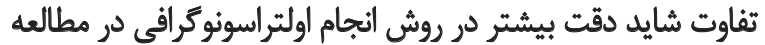

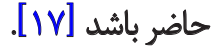

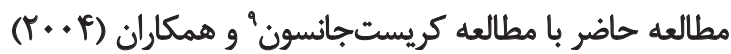
كه در بررسى تكراريذيرى اولتراسونوكرافى در اندازهيرى سطي سطح

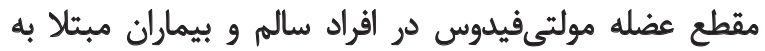

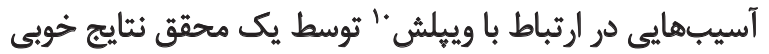

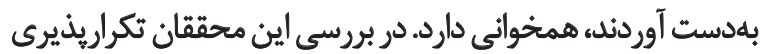

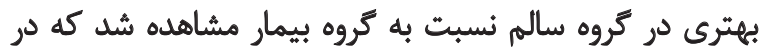

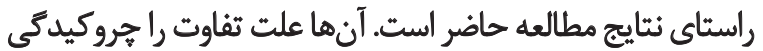

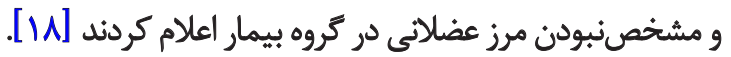
مطالعانى كه در اين زمينه صورت ترفته فقط تكراريذيرى

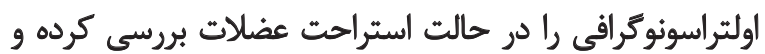

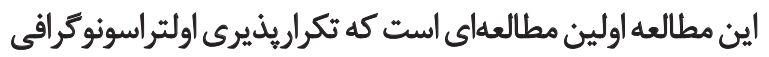

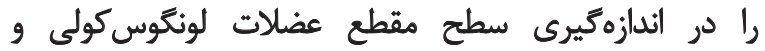

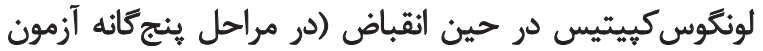

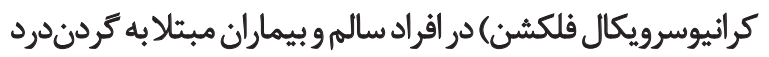

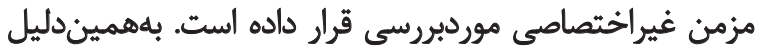

امكان مقايسه نتايج اين مطالعه با مطالعات ديكّر وجود ندارد. بردي

\section{نتيجهُيرى}

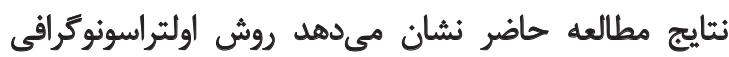

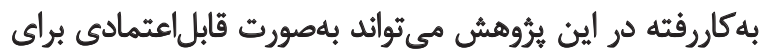

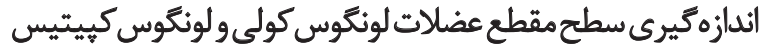

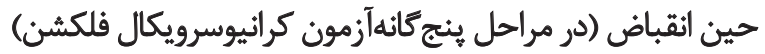

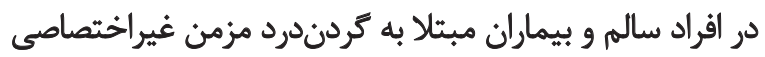

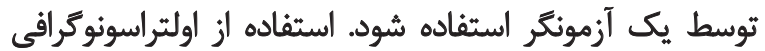

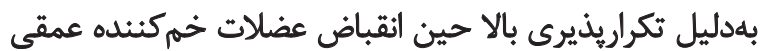

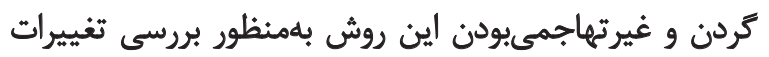

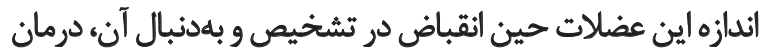

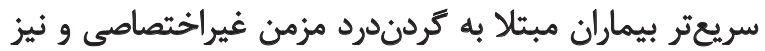

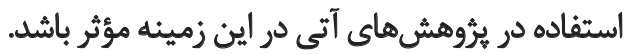

محدوديتها و يشئنهاداث

ازآنجاكه مطالعه حاضر روى دانشجويان و كاركنان دانشعاه علوم بهزيستى و توانبخشى انجام كرفت و به ائ اين جامعانه جوان كوان

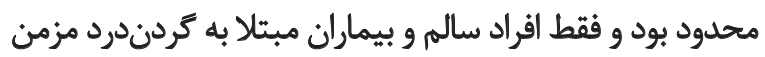




\section{References}

[1] Hodges PW, Richardson CA. Inefficient muscular stabilization of the lumbar spine associated with low back pain: a motor control evaluation of transversus abdominis. Spine. 1996; 21(22):2640-50. PMID: 8961451

[2] Whittaker JL, Teyhen DS, Elliott JM, Cook K, Langevin HM, Dahl $\mathrm{HH}$, et al. Rehabilitative ultrasound imaging: understanding the technology and its applications. Journal of Orthopaedic \& Sports Physical Therapy. 2007; 37(8):434-49. doi: 10.2519/ jospt.2007.2350

[3] Emshoff R, Bertram S, Strobl H. Ultrasonographic cross-sectional characteristics of muscles of the head and neck. Oral Surgery, Oral Medicine, Oral Pathology, Oral Radiology, and Endodontology. 1999; 87(1):93-106. doi: 10.1016/s1079-2104(99)70302-1

[4] Neumann DA. Kinesiology of the musculoskeletal system: foundations for rehabilitation. $2^{\text {nd }} \mathrm{ed}$. Mosby: Elsevier Health Sciences; 2009

[5] Oatis C. Kinesiology: The mechanics \& pathomechanics of human movement. Philadelphia: Williams \& Wilkins Publication; 2004

[6] Jull G, Kristjansson E, Dall'Alba P. Impairment in the cervical flexors: a comparison of whiplash and insidious onset neck pain patients. Manual Therapy. 2004; 9(2):89-94. doi: 10.1016/s1356689x(03)00086-9

[7] Falla D, Jull G, Dall'Alba P, Rainoldi A, Merletti R. An electromyographic analysis of the deep cervical flexor muscles in performance of craniocervical flexion. Physical Therapy. 2003; 83(10):899-906. doi: 10.1093/ptj/83.10.899

[8] Rankin G, Stokes M, Newham D. Size and shape of the posterior neck muscles measured by ultrasound imaging: normal values in males and females of different ages. Manual Therapy. 2005; 10(2):108-15. doi: 10.1016/j.math.2004.08.004

[9] Dickx N, Cagnie B, Achten E, Vandemaele P, Parlevliet T, Danneels L. Differentiation between deep and superficial fibers of the lumbar multifidus by magnetic resonance imaging. European Spine Journal. 2010; 19(1):122-8. doi: 10.1007/s00586-0091171-x

[10] Javanshir K, Mohseni-Bandpei MA, Rezasoltani A, Amiri M, Rahgozar M. Ultrasonography of longus colli muscle: a reliability study on healthy subjects and patients with chronic neck pain. Journal of Bodywork and Movement Therapies. 2011; 15(1):50-6. doi: 10.1016/j.jbmt.2009.07.005

[11] Rezasoltani A. The applicability of muscle ultrasonography in physiotherapy researches. Journal of Physical Therapy Science. 2003; 15(1):33-7. doi: 10.1589/jpts.15.33

[12] Rezasoltani A, Ylinen J, Vihko V. Isometric cervical extension force and dimensions of semispinalis capitis muscle. Journal of Rehabilitation Research and Development. 2002; 39(3):423-8.

[13] Javanshir K, Rezasoltani A, Mohseni-Bandpei MA, Amiri M, Ortega-Santiago R, Fernández-de-las-Penas C. Ultrasound assessment of bilateral longus colli muscles in subjects with chronic bilateral neck pain. American Journal of Physical Medicine \& Rehabilitation. 2011; 90(4):293-301. doi: 10.1097/ phm.0b013e31820173e5
[14] Stokes M, Rankin G, Newham D. Ultrasound imaging of lumbar multifidus muscle: normal reference ranges for measurements and practical guidance on the technique. Manual Therapy. 2005; 10(2):116-26. doi: 10.1016/j.math.2004.08.013

[15] Jull GA, O'Leary SP, Falla DL. Clinical assessment of the deep cervical flexor muscles: the craniocervical flexion test. Journal of Manipulative and Physiological Therapeutics. 2008; 31(7):525-33. doi: 10.1016/j.jmpt.2008.08.003

[16] Rosner B. Fundamentals of biostatistics. Belmont: Thomson Brooks; 2006.

[17] Cagnie B, Derese E, Vandamme L, Verstraete K, Cambier D, Danneels L. Validity and reliability of ultrasonography for the longus colli in asymptomatic subjects. Manual Therapy. 2009; 14(4):421-6.

[18] Kristjansson E. Reliability of ultrasonography for the cervical multifidus muscle in asymptomatic and symptomatic subjects. Manual Therapy. 2004; 9(2):83-8. doi: 10.1016/s1356689x(03)00059-6 
